\title{
In Vivo Curative and Antacid Effects of Cameroonian Clay (MY41g) on Chronic and "Unhealed" Gastric Ulcers in Rats
}

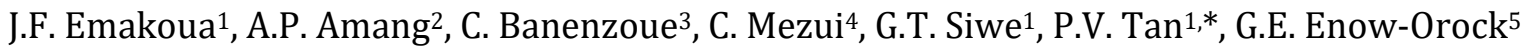 \\ ${ }^{1}$ Department of Animal Biology \& Physiology, Faculty of Science, University of Yaounde I, Yaounde, P.O. Box 812, Cameroon. \\ ${ }^{2}$ Department of Biological Sciences, Faculty of Science, University of Maroua, Maroua, P.O. Box 814, Cameroon. \\ ${ }^{3}$ Department of Inorganic Chemistry, Faculty of Science, University of Douala, Douala, P.O. Box 24 157 Cameroon. \\ ${ }^{4}$ Department of Biological Sciences, Higher Teachers' Training College, University of Yaounde I, Yaounde, P.O. Box 047, Cameroon. \\ ${ }^{5}$ Department of Biomedical Sciences, Faculty of Health Sciences, University of Buea, Buea, P.O. Box 63, Cameroon.
}

\section{ART I C LE DETA IL S}

\section{Article history:}

Received 21 July 2020

Accepted 08 August 2020

Available online 13 September 2020

\section{Keywords:}

MY41g Clay

Chronic Gastric Ulcers

Unhealed Ulcers

Antacid Potential

\begin{abstract}
A B S T R A C T
This study evaluated the in vivo curative and antacid effects of MY41g clay on chronic and "unhealed" gastric ulcers in rats. Chronic gastric ulcers were induced by injecting $0.05 \mathrm{~mL}$ of acetic acid (30\%) into the stomach wall. From day 5-14 after induction of ulcers, rats were treated daily with MY41g clay (125 and $250 \mathrm{mg} / \mathrm{kg}$ ). For "Unhealed" gastric ulcers, from day 5-18 rats received MY41g clay orally concomitantly with indomethacin $(1 \mathrm{mg} / \mathrm{kg} /$ day $)$ subcutaneously. The ulcer index, percentage of healing, mucus secretion, histological parameters, oxidative stress parameters and gastric acidity were assessed. Treatment with clay solution for 10 days resulted in accelerated spontaneous healing of chronic gastric ulcers (83.69-90.2\%). However, indomethacin administration did not induce significant variations in the percentage of healing (89.23-91.66\%) in rats. For both ulcer models performed, ulcer healing was accompanied by a significant increase $(\mathrm{p}<0.001)$ of mucus secretion at the highest dose. Clay increased concentrations of antioxidant enzymes and decreased gastric acidity and lipid peroxidation. Administration of clay accelerated the spontaneous healing of both induction models. The mode of action of the clay could involve increased gastric mucus production, gastric mucosal re-epithelialization, improved antioxidant status and gastric acid neutralization. MY41g clay can be used as antacids in the ulcer treatment regime.
\end{abstract}

\section{Introduction}

The problem of treating gastric ulcers in underdeveloped countries remains a major concern due to poverty, the inadequacy of modern health infrastructures and the very high cost of conventional triple therapy as well as the associated side effects [1]. Thus, most of the affected persons in these countries are using traditional medicine. Traditional medication uses medicinal plants, animal parts and minerals to cope with gastric ulcers [1]. While many studies have shown the anti-ulcer properties of medicinal plants, very little has been devoted to mineral sources such as clay. Clay represents different sedimentary rocks with a high mineral content. The structures and properties of clays therefore vary according to their mineral composition and concentration [2]. For example, smectites represent a family of clays containing montmorillonites, bentonites, saponites, nontronites and beidellites [3]. This family of clays is known for its ability to trap water molecules and to fix cations to form a gel that is a good dressing for the digestive tract [4].

A WHO study in 2002 [5] demonstrated the curative effect of clay against Buruli ulcer. Clays are found in pharmacies as drugs for the treatment of certain digestive diseases. The modes of action of some claybased products have been elucidated: Bedelix (smectite beidelleitic clay) for the symptomatic treatment of irritable colon syndrome; Gelox (smectite clay) for the symptomatic treatment of painful manifestations during oesophageal-gastro-duodenal disorders; Smecta (smectite clay) for the treatment of acute and chronic diarrhoea, symptomatic treatment of pain related to oesophageal-gastro-duodenal and colic disorders; Kaologeais (kaolinite clay) for the symptomatic treatment of digestive functional disorders accompanied by anxiety symptoms [6].

Cameroon has large clayey deposits, particularly kaolinite and halloysite. Cameroonian clays are consumed by geophagia; as antibiotics for wounds, as detoxifyer, as antidiarrhetics, as antiemetics in pregnant women and as antacids against gastric ulcers [7]. The valorization of these clays in the pharmacological field could open up other ways of using these resources. Preliminary in vitro work carried out by Banenzoue et al. [8] on clays from the West region of Cameroon showed that the Mayouom clay sample (MY41g) when combined with $2 \%$ calcium carbonate, had maximal antacid capacity at an inclusion rate of $2.5 \mathrm{~g}$. The central role of gastric acid hypersecretion in the etiology of peptic ulcers is well known [9], and the control of gastric acidity is a cornerstone for promoting ulcer healing [10]. Hence our interest in studying the antacid and ulcer healing effects in vivo of the MY41g clay sample on chronic gastric ulcers in rats. In some cases, ulcer healing may be delayed as in elderly patients routinely using non-steroidal anti-inflammatory drugs to relieve the pain induced by other age-related conditions. For these reasons, the aim of our study was to evaluate the antacid and curative effects in vivo of the MY41g clay sample of Cameroonian origin on both simple chronic and "unhealed" gastric ulcers. Simple chronic gastric ulcers were induced in experimental animals using glacial acetic acid, and "unhealed" chronic ulcers were produced by associating a non-steroidal anti-inflammatory drug indomethacin

\section{Experimental Methods}

\subsection{Material}

\subsubsection{Geological Material}

The MY41g clay and limestone used in this experiment were obtained, respectively, from the Mayouom clay deposit in the Noun Division, West Region of Cameroon, and the Figuil limestone deposit in the Mayo Louti Division, North Region of Cameroon [11]. After harvesting, they were crushed in a mortar into a fine powder and passed through a sieve. Only the particles that passed through the one nanometer sieve pore diameter were used in this study. 


\subsubsection{Experimental Animals}

The animals used were male albino rats of the Wistar strain (Rattus norvegicus), aged 12 to 14 weeks and with body weights between $150 \mathrm{~g}$ and $200 \mathrm{~g}$. The rats were raised in the Animal house of the Animal Physiology Laboratory, Department of Animal Biology and Physiology of the University of Yaoundé I. They were kept at room temperature under natural day/night cycles, fed with a standard laboratory diet (supplied by SPC Ltd, Bafoussam, Cameroon) and given tap water ad libitum. Prior authorization for the use of laboratory animals in this study was obtained from the Cameroon National Ethics Committee (registration number: FWA-IRB00001954), which permits, among other procedures, the use of ether anesthesia for animal research. Otherwise, the use, handling, and care of animals were done in adherence to the European Convention for the Protection of Vertebrate Animals Used for Experimental and Other Purposes (ETS-123), with particular attention to Part III, articles 7, 8, and 9 [12].

\subsection{Methods}

\subsubsection{Preparation of Clay Solution}

$2.4 \mathrm{~g}$ of clay powder was mixed with $0.1 \mathrm{~g}$ of limestone and $50 \mathrm{~mL}$ of distilled water. The mixture was homogenized using a magnetic stirrer to obtain a stock solution with a concentration of $50 \mathrm{mg} / \mathrm{mL}$.

\subsubsection{Induction of Gastric Ulcers}

\subsubsection{Induction of Simple Chronic Acetic Acid Ulcers}

The induction of chronic gastric ulcers was performed according to the method described by Pillai and Santhakumari [13].

After 24 hours of non-hydric fasting, 30 rats were divided into 6 groups of 5 animals each. Under ether anesthesia, an abdominal incision was made. A volume of $0.05 \mathrm{~mL}$ of glacial acetic acid (30\%) was injected into the stomach wall at the small curvature. After cleaning the stomach with cotton soaked in $\mathrm{NaCl}$ solution (9\%), a suture was performed to close the incision. An antibiotic (Betadine) was applied to the incision to prevent infection of the wound.

Three days after ulcer induction, group 1 rats were fasted for 24 hours, the incisions re-opened and the pylorus of each rat was ligated according to the method described by Hara and Okabe [14]. These rats were sacrificed 6 hours later under anesthesia, and the rat stomachs were opened in order to establish the degree of ulceration prior to the onset of treatment. From the $5^{\text {th }}$ day after injection with acetic acid, groups $(2,3,4$, and 5) were treated daily by gavage for 10 days as follows: group 2 rats (longitudinal control) received $1 \mathrm{~mL} / 200 \mathrm{~g}$ distilled water; group 3 and 4 rats received MY41g clay solution at 125 and $250 \mathrm{mg} / \mathrm{kg}$, respectively; group 5 rats received $50 \mathrm{mg} / \mathrm{kg}$ sucralfate. On the $9^{\text {th }}$ day of treatment, the animals were fasted for 24 hours. The next day, 30 minutes after the last dose of treatment, the incisions were re-opened, the pylorus of each rat ligated, and the abdomens re-sutured. The rats were sacrificed 6 hours later under anesthesia, and then underwent the same protocol as the animals sacrificed 4 days after ulcer induction.

\subsubsection{Induction of "Unhealed" Gastric Ulcers}

The method described by Pillai and Santhakumari in 1984 was used and supplemented by that of Wang [15] with some modifications: From the $5^{\text {th }}$ day after induction of chronic gastric ulcers, rats in groups $2,3,4$ and 5 were given indomethacin ( $1 \mathrm{mg} / \mathrm{kg} /$ day) subcutaneously 30 minutes before each clay treatment; the treatment lasted for 14 days.

\subsubsection{Measurement of Mucus Production}

The mucus on the glandular part of the stomach of each rat was gently scraped off using a microscope slide [16], and weighed using a sensitive electronic balance.

\subsubsection{Measurement of Gastric Acidity}

The gastric juice collected from each rat was centrifuged at $4000 \mathrm{rpm}$ for 10 minutes to remove residual debris. $1 \mathrm{~mL}$ of this centrifuged juice was used to determine the hydrogen ion concentration by $\mathrm{pH}$-metric titration against a $0.1 \mathrm{~N} \mathrm{NaOH}$ solution using a digital $\mathrm{pH}$ meter. The acid concentration was expressed in meq./L [17].

\subsubsection{Preparation of Histological Sections}

Sections of stomach walls were made perpendicular to the surface of each ulcer crater. Sections of the normal stomach were also made for comparison. The haematoxylin-eosin (H\&E) staining technique was used according to the standard histological procedure described by BayeletVincent [18] and the sections were observed microscopically. https://doi.org/10.30799/jpmr.048.20050103

\subsubsection{Measurement of In Vivo Antioxidant Capacity}

Oxidative stress parameters were measured on supernatant of crushed stomach samples after centrifuging at $5700 \mathrm{rpm}$ for $10 \mathrm{~min}$. Total protein was determined using the Biuret method [19]. Cellular glutathione (GSH) was measured on the basis of the reaction between 2,2-dithio-5,5dibenzoic acid and the thiol ( $\mathrm{SH}$ ) groups of glutathione to give a complex whose absorbance was read at $412 \mathrm{~nm}$ [20]. The glutathione concentration was calculated using the molar extinction coefficient $\varepsilon=$ $1.36104 \mathrm{M}^{-1} \mathrm{~cm}^{-1}$. Superoxide dismutase (SOD) activity was measured using a standard method [21], while catalase was determined and expressed in $\mathrm{mM}$ of $\mathrm{H}_{2} \mathrm{O}_{2} / \mathrm{min} / \mathrm{mg}$ protein [22]. Lipid peroxidation was assessed by measuring malondialdehyde (MDA) levels in gastric tissue samples [23]. The quantification of the MDA was performed using an extinction coefficient of $=1.56 . \times 105 \mathrm{M}^{-1} \mathrm{~cm}^{-1}$.

\subsubsection{Statistical Analysis}

Significant differences between the means of the treatment groups were determined by the analysis of variance (one-way ANOVA) followed by the Tukey multiple comparison test. Values of $p<0.05$ were considered significant. The results were expressed as arithmetic means \pm standard error of the mean (S.E.M.).

\section{Results and Discussion}

Fig. 1 show macroscopic photographs of the stomachs of the rats from different treatment groups after induction of simple chronic gastric ulcers. Fig. 1(a) shows the stomach of a normal rat without ulcer. The stomachs of rats sacrificed 4 days after ulcer induction had deep and wide ulcer craters with raised edges and sclerous interior, representing an ulceration surface of $72.00 \mathrm{~mm}^{2}$ (Fig. (1b)). The treatment of the ulcers with distilled water for 10 days (longitudinal control) resulted in a reduction of the ulcerated areas to $20.75 \mathrm{~mm}^{2}$, representing an auto-healing rate of $71.18 \%$ (Fig. 1(c).

Treatment of ulcerated rats with MY41g at 125 and $250 \mathrm{mg} / \mathrm{kg}$ for 10 days resulted in a significant decrease $(\mathrm{p}<0.01$ and $\mathrm{p}<0.001$, respectively) in the ulcerated areas $\left(11.75 \mathrm{~mm}^{2}\right.$ and $7.00 \mathrm{~mm}^{2}$, respectively) (Figs. 1(d) and (e)) compared to the longitudinal control; corresponding to a healing rate of 83.69 and $90.20 \%$, respectively. This healing was accompanied by a significant increase $(\mathrm{p}<0.001)$ in mucus secretion from $92.50 \mathrm{mg}$ in the longitudinal control to $160.30 \mathrm{mg}$ in animals treated with MY41g clay at the $250 \mathrm{mg} / \mathrm{kg}$ dose. For rats treated with sucralfate, the significant decrease $(\mathrm{p}<0.001)$ in the ulcerated area $\left(0.50 \mathrm{~mm}^{2}\right.$, (Fig. 1(f) healing rate, $99.30 \%)$ was also accompanied by a significant increase $(\mathrm{p}<0.001)$ in mucus production (152.3 $\mathrm{mg}$ ) compared to the longitudinal control (20.75 mg) (Table 1). Treatment with the MY41g clay solution caused a significant decrease $(\mathrm{p}<0.001)$ in gastric acidity at 125 and $250 \mathrm{mg} / \mathrm{kg}$ doses compared to the cross-sectional control.

Indeed, gastric $\mathrm{pH}$ and acidity increased from $2.41 \pm 0.23$ in the crosssectional controls to $5.45 \pm 0.66$ and $5.83 \pm 0.32$ in rats treated with 125 and $250 \mathrm{mg} / \mathrm{kg}$ MY41g clay, respectively $(\mathrm{p}<0.01)$. Corresponding values for gastric acidity dropped progressively from 73.75 meq./L in the crosssectional controls to 35.25 and 25,44 meq./L for clay-treated groups (p < 0.001 ). Both doses of clay treatment were more efficient than Sucralfate in reducing gastric acidity (Table 2 ).

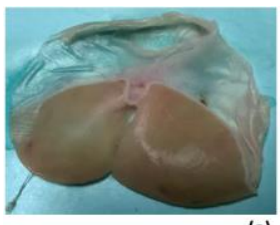

(a)

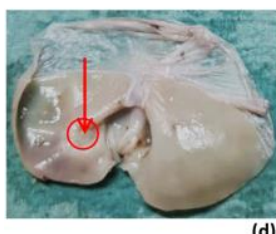

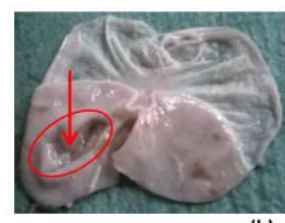

(b)

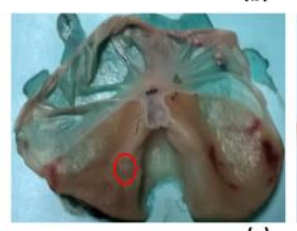

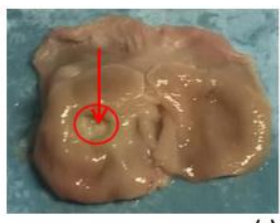

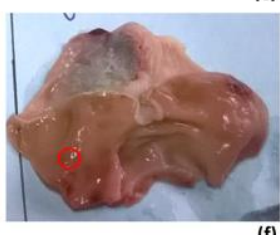

Fig. 1 Stomachs of rats with simple acetic acid-induced chronic gastric ulcers. 1(a): Normal rat (untreated, non-ulcerated rats that received distilled water for 10 days); 1(b): Group 1 rats sacrificed 4 days after induction of chronic gastric ulcers to confirm ulcer formation; 1 (c): Longitudinal control/group 2 rats that received daily distilled water $(1 \mathrm{~mL} / 200 \mathrm{~g})$ for 10 days from the 5 th day after induction of chronic gastric ulcers; $1 \mathrm{~d}$ and e: group $3+4$ rats treated with 125 and $250 \mathrm{mg} / \mathrm{kg}$ MY41g clay solution; 1(f) : group 5 ulcerated rats given $50 \mathrm{mg} / \mathrm{kg}$ sucralfate (reference drug) for 10 days from the 5 th day after induction of chronic gastric ulcers); $€$ indication of chronic gastric ulcers. 
Table 1 Effects of MY41g clay on simple acetic acid-induced chronic gastric ulcers

\begin{tabular}{|c|c|c|c|c|c|c|}
\hline Treatment & Dose (mg/kg) & $\mathrm{N}$ & Ulcer index (IU) & $\%$ ulcerated area & \% Healing & Mucus production (mg) \\
\hline Control 1 & - & 5 & $72.00 \pm 0.81$ & 10.66 & - & $54.25 \pm 0.62$ \\
\hline Control 2 & _ & 5 & $20.75 \pm 1.10^{* * *}$ & 3.07 & 71.18(s-h) & $92.50 \pm 3.797^{*}$ \\
\hline MY41g & 125 & 5 & $11.75 \pm 1.181^{* * * \# \#}$ & 1.74 & 83.69 & $27.75 \pm 3.816 \# \# \#$ \\
\hline MY41g & 250 & 5 & 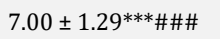 & 0.10 & 90.27 & $160.3 \pm 11.92^{* * *} \# \# \#$ \\
\hline Sucralfate & 50 & 5 & 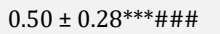 & 0.07 & 99.30 & $152.3 \pm 9.132^{* * *} \# \# \#$ \\
\hline
\end{tabular}

Control 1 (4 day ulcerated rats); Control 2 (spontaneous healing); $N=$ number of rats; the values in the table represent averages \pm ESM; $(s-h)=\operatorname{self-healing;~} p<0.05 ;{ }^{* *} p<0.01 ;$ and

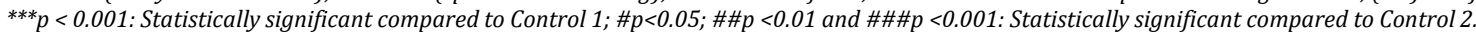

Table 2 Effects of MY41g clay on gastric $\mathrm{pH}$ in rats with simple chronic gastric ulcers

\begin{tabular}{|c|c|c|c|c|}
\hline Treatment & Dose (mg/kg) & $\mathrm{N}$ & Gastric pH & Gastric acidity (meq./L) \\
\hline Control 1 & - & 5 & $2.41 \pm 0.23$ & $73.75 \pm 3.45$ \\
\hline Control 2 & - & 5 & $4.45 \pm 0.38^{* *}$ & $42.50 \pm 1.07^{* * *}$ \\
\hline MY41g & 125 & 5 & $5.45 \pm 0.66^{* * *}$ & $35.25 \pm 0.51^{* * *}$ \\
\hline MY41g & 250 & 5 & $5.83 \pm 0.32^{* * * \#}$ & $25.44 \pm 1.78^{* * * \# \#}$ \\
\hline Sucralfate & 50 & 5 & $3.09 \pm 0.31^{\#}$ & $53.75 \pm 0.71^{* * \#}$ \\
\hline
\end{tabular}

Control 1 (4 day ulcerated rats); Control 2 (spontaneous healing); $N=$ number of rats; the values in the table represent averages \pm ESM; ${ }^{*} p<0.05 ;{ }^{* *} p<0.01 ;$ and ${ }^{* * *} p<0.001$ :

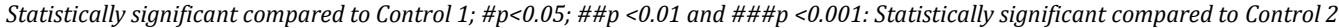

Table 3 Effects of MY41g clay on tissue oxidative stress parameters in rats with simple chronic gastric ulcers

\begin{tabular}{|c|c|c|c|c|c|c|}
\hline Treatment & $\begin{array}{l}\text { Dose } \\
(\mathrm{mg} / \mathrm{kg})\end{array}$ & $\mathrm{N}$ & $\begin{array}{l}\text { SOD } \\
\text { (U/mg protein) }\end{array}$ & $\begin{array}{l}\text { Catalase } \\
\left(\mu \text { mol } \mathrm{H}_{2} \mathrm{O}_{2} / \mathrm{min} / \mathrm{mg} \text { protein }\right)\end{array}$ & $\begin{array}{l}\text { GSH } \\
\text { (mmol/g protein) }\end{array}$ & $\begin{array}{l}\text { Malondi aldehyde } \\
\text { (pmol/mg protein) }\end{array}$ \\
\hline Normal Rats & - & 5 & $2.48 \pm 0.17$ & $5.53 \pm 1.25$ & $1.68 \pm 0.32$ & $5.35 \pm 0.50$ \\
\hline Control 1 & - & 5 & $1.50 \pm 0.15$ & $8.35 \pm 0.65$ & $3.23 \pm 0.03$ & $9.60 \pm 0.33$ \\
\hline Control 2 & _ & 5 & $1.58 \pm 0.11$ & $8.50 \pm 0.29$ & $2.85 \pm 0.20$ & $8.92 \pm 0.51$ \\
\hline MY41g & 125 & 5 & $3.19 \pm 0.42^{* * \# \#}$ & $5.12 \pm 0.23^{* * \# \#}$ & $4.43 \pm 0.22^{\#}$ & $6.18 \pm 3.34$ **\#\# \\
\hline MY41g & 250 & 5 & $5.55 \pm 0.43^{* * * \# \# \#}$ & $8.21 \pm 0.50$ & $3.44 \pm 0.21$ & $7.63 \pm 0.24^{\#}$ \\
\hline Sucralfate & 50 & 5 & $1.67 \pm 0.17$ & $8.45 \pm 1.55$ & $2.43 \pm 0.23$ & $7.85 \pm 0.15$ \\
\hline
\end{tabular}

Control 1 (4 day ulcerated rats); Control 2 (spontaneous healing); $N=$ number of rats; the values in the table represent averages \pm ESM; ${ }^{*}<<0.05 ;{ }^{* *} p<0.01 ;$ and ${ }^{* * *} p<0.001$ :

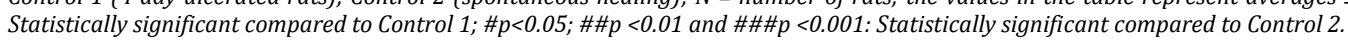

Fig. 2 shows the histological sections of the stomachs of the rats with simple chronic gastric ulcers induced with acetic acid. In comparison with the normal gastric mucosa in non-ulcerated rats (Fig. 2(a)) day 4 of ulceration created deep ulcer craters with superficial loss of substance, glandular destruction, fibrosis, sclerosis and edema (Fig. 2(b)). Rats in the spontaneous healing group show an ulcerated area invaded by inflammatory cells, an onset of glandular recovery with regression of edema but with persistence of fibrosis (Fig. 2(c)). Rats treated with 125 and $250 \mathrm{mg} / \mathrm{kg} \mathrm{MY41g} \mathrm{clay} \mathrm{had} \mathrm{glandular} \mathrm{proliferation,} \mathrm{with} \mathrm{near} \mathrm{total}$ recovery of the gastric glands and with disappearance of fibrosis and edema (Figs. 2(d) and (e)). Sucralfate treatment resulted in marked healing but with slight persistence of the destroyed mucosa (Fig. 2(f)).

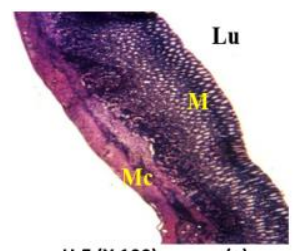

H.E (X 100) (a)
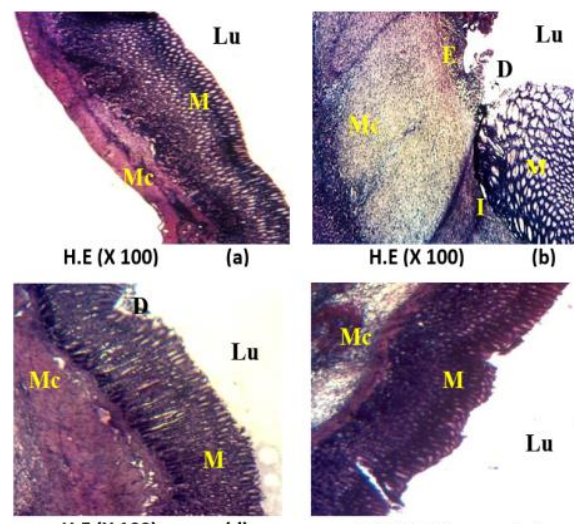

H.E (X 100)

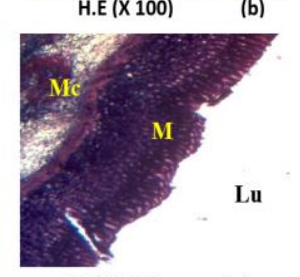

H.E (X 100)
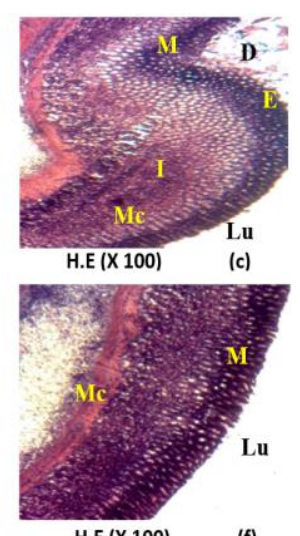

H.E (X 100) (f)
Fig. 2 Histological presentation of simple chronic ulcers in rats. 1(a) : normal rat (with normal mucosa and sub mucosa); 1(b) : control 1 (with deep ulcers, with superficial loss of substance and glandular destruction down to the sub mucosa); 1 (c) : Longitudinal control (ulcerated area invaded by inflammatory cells, with onset of glandular recovery; 1(d and e): Rats receiving 125 and $250 \mathrm{mg} / \mathrm{kg}$ of MY41g clay, with glandular proliferation, leukocyte infiltration and partial recovery of the ulcerated area; 1(f) : Sucralfate-treated stomach with healing, and a slight persistence of the destroyed mucosa; D: destruction; E: edema; H.E: HematoxylinEosin; I: Leukocyte infiltration; Lu: Gastric lumen; M: mucosa; Mc: Muscle layer

Table 3 shows oxidative stress parameters of rats with simple chronic ulcers. Acetic ulceration caused SOD activity to drop to $1.5-1.58 \mathrm{U} / \mathrm{mg}$ protein compared to 2.48 in normal rats, but clay treatment raised SOD activity to above normal levels (3.19-5.55 U/mg protein). Sucralfate did not have a similar effect. Gastric ulceration increased MDA concentrations to $8.9-9.6 \mathrm{pmol} / \mathrm{mg}$ protein compared to normal rats $(5.35 \mathrm{pmol} / \mathrm{mg}$ protein), but clay treatment dropped MDA values to $6.18 \mathrm{pmol} / \mathrm{mg}$ protein at the dose of $125 \mathrm{mg} / \mathrm{kg}$.

https://doi.org/10.30799/jpmr.048.20050103
Fig. 3 shows the macroscopic aspects of the stomachs of rats subjected to the unhealed gastric ulcers: (chronic ulcers + indomethacin administration for 2 weeks). Figs. 3(a) and (b) show the stomachs of a normal rat and a group 1 rat (day 4 after ulceration) similar to those described above. Administration of indomethacin to ulcerated rats for 14 days further widened and deepened the ulcer wound, giving an ulcer index of $37.50 \pm 1,44$ and healing $\%$ of 47,91 (Fig. 3(d)). Photo 3(c) shows the stomach of ulcerated rat in the simple longitudinal control group given water for 14 days without indomethacin. A reduction in crater depth with mucus deposition on the surface of the ulcer and hemorrhagic ulcer boundaries: with $3.62 \%$ ulcerated surface and self-healing of $65.97 \%$ (Table 4). Figs. 3(e) and (f) represent the stomachs of ulcerated rats treated with MY41g clay at 125 and $250 \mathrm{mg} / \mathrm{kg}$, respectively; ulcer craters reduced to 1.14 and $0.88 \%$ of the total glandular area respectively, with a lower mucus deposition (71.25 and $72.25 \mathrm{mg}$, respectively) compared to the spontaneous healing in ulcerated rats without indomethacin (142 mg).

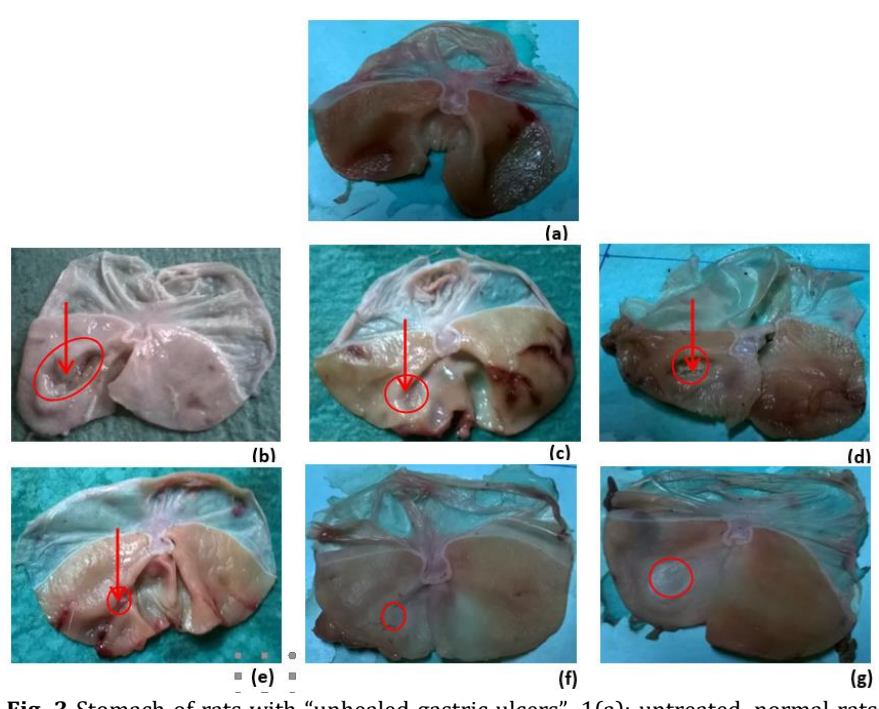

Fig. 3 Stomach of rats with "unhealed gastric ulcers". 1(a): untreated, normal rats that received distilled water for 14 days; 1 (b): Ulcerated rats sacrificed 4 days after induction of chronic gastric ulcers to confirm ulcer formation; (c): rats that received daily distilled water $(1 \mathrm{~mL} / 200 \mathrm{~g})$ for 14 days from the 5 th day after induction of chronic gastric ulcers 1 (d): Ulcerated rats that received daily distilled water (1 $\mathrm{mL} / 200 \mathrm{~g})+$ indomethacin $(1 \mathrm{mg} / \mathrm{kg})$ for 14 days from the $5^{\text {th }}$ day after induction of chronic gastric ulcers; (e and $\mathrm{f}$ ): rats that received MY41g clay solution + indomethacin $(1 \mathrm{mg} / \mathrm{kg})$ for 14 days from the $5^{\text {th }}$ day after induction of ulcers; $(\mathrm{g})$ : rats that received sucralfate daily + indomethacin $(1 \mathrm{mg} / \mathrm{kg})$ for 14 days from the $5^{\text {th }}$ day after induction of ulcers; indication of gastric ulcers. 
Table 4 Effects of MY41g clay on "unhealed gastric ulcers"

\begin{tabular}{|c|c|c|c|c|c|c|}
\hline Treatment & Dose (mg/kg) & $\mathrm{N}$ & Ulcer index (IU) & $\%$ ulcerated area & (\%) Healing & Mucus production (mg) \\
\hline Control 1 & - & 5 & $72.00 \pm 0.81$ & 10.66 & - & $54.25 \pm 0.62$ \\
\hline Control 2 & - & 5 & $24.50 \pm 2.06^{* * *}$ & 3.62 & $65.97(s-h)$ & $142.00 \pm 3.93^{* * *} \varphi \varphi \varphi$ \\
\hline Control 3 & - & 5 & $37.50 \pm 1.44^{* * *}$ & 5.55 & $47.91(\mathrm{~s}-\mathrm{h})$ & $71.75 \pm 1.84$ \\
\hline MY41g & 125 & 5 & $7.75 \pm 0.62^{* * *} \# \# \varphi \varphi \varphi$ & 1.14 & 89.23 & $71.25 \pm 7.77 \# \# \#$ \\
\hline MY41g & 250 & 5 & $6.00 \pm 0.81^{* * *} \# \# \# \varphi \varphi \varphi$ & 0.88 & 91.66 & $72.25 \pm 2.78 \# \# \#$ \\
\hline Sucralfate & 50 & 5 & $4.75 \pm 0.47^{* * *} \# \# \# \varphi \varphi \varphi$ & 0.70 & 93.40 & $73.00 \pm 3.62 * \# \# \#$ \\
\hline
\end{tabular}

Control 1 (4 day ulcerated rats); Control 2: (spontaneous healing in ulcerated rats without indomethacin) Control 3: (spontaneous healing in ulcerated rats given indomethacin). $N=$ number of rats; the values in the table represent averages $\pm E S M ;(s-h)=$ self-healing; ${ }^{*} p<0.05 ;{ }^{* *} p<0.01 ;$ and ${ }^{* * *} p<0.001:$ Statistically significant compared to Control $1 ;$

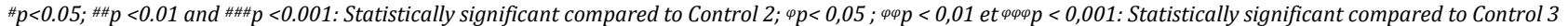

Table 5 Effects of MY41g clay on gastric $\mathrm{pH}$ in rats subjected to "unhealed gastric ulcers"

\begin{tabular}{|c|c|c|c|c|}
\hline Treatment & Dose (mg/kg) & $\mathrm{N}$ & Gastric pH & Gastric acidity (meq./L) \\
\hline Control 1 & _ & 5 & $2.41 \pm 0.23$ & $73.75 \pm 3.45$ \\
\hline Control 2 & - & 5 & $3.59 \pm 0.23^{*}$ & $48.24 \pm 1.44^{* * *}$ \\
\hline Control 3 & - & 5 & $2.41 \pm 0.21$ & $82.38 \pm 4.93$ \\
\hline MY41g & 125 & 5 & $5.33 \pm 0.38^{* * * \# \# \varphi \varphi \varphi}$ & $36.25 \pm 2.14^{* * *} \varphi \varphi \varphi$ \\
\hline MY41g & 250 & 5 & 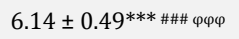 & $21.25 \pm 1.13^{* * * \# \# \varphi \varphi \varphi}$ \\
\hline Sucralfate & 50 & 5 & $5.55 \pm 0.20^{* * * \# \# \varphi \varphi \varphi}$ & $26.81 \pm 0.52^{* * * \# \# \varphi \varphi \varphi}$ \\
\hline
\end{tabular}

Control 1 (4 day ulcerated rats); Control 2: (spontaneous healing in ulcerated rats without indomethacin) Control 3: (spontaneous healing in ulcerated rats given indomethacin). $N=$ number of rats; the values in the table represent averages $\pm E S M ;{ }^{*} p<0.05 ;{ }^{* *} p<0.01 ;$ and ${ }^{* * *} p<0.001$ : Statistically significant compared to Control $1 ;{ }^{*} p<0.05 ; \# \#<<0.01$ and

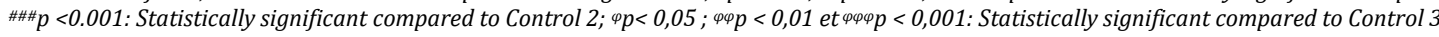

Table 6 Effects of MY41g clay on tissue oxidative stress parameters in rats with "unhealed gastric ulcers"

\begin{tabular}{|c|c|c|c|c|c|c|}
\hline Treatment & Dose (mg/kg) & $\mathrm{N}$ & $\begin{array}{l}\text { SOD }(\mathrm{U} / \mathrm{mg} \\
\text { protein) }\end{array}$ & $\begin{array}{l}\text { Catalase }\left(\mu \mathrm{mol} \mathrm{H} \mathrm{O}_{2} / \mathrm{min} / \mathrm{mg}\right. \\
\text { protein })\end{array}$ & GSH (mmol/g protein) & $\begin{array}{l}\text { Malondialdehyde } \\
\text { (pmol/mg protein) }\end{array}$ \\
\hline Normal Rats & - & 5 & $2.48 \pm 0.17$ & $5.53 \pm 1.25$ & $1.68 \pm 0.32$ & $5.35 \pm 0.50$ \\
\hline Control 1 & - & 5 & $1.50 \pm 0.15$ & $8.35 \pm 0.65$ & $3.23 \pm 0.03$ & $9.60 \pm 0.33$ \\
\hline Control 2 & - & 5 & $1.90 \pm 0.37$ & $6.44 \pm 0.75^{*} \varphi$ & $2.20 \pm 0.27^{*}$ & $14.11 \pm 1.73^{* *}$ \\
\hline Control 3 & - & 5 & $1.06 \pm 0.19$ & $8.25 \pm 0.08$ & $2.14 \pm 0.01^{*}$ & $13.20 \pm 1.56^{* *}$ \\
\hline MY41g + Indo & 125 & 5 & $2.2 \pm 0.06$ & 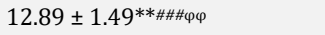 & $5.23 \pm 0.48^{* \# \# \# \varphi \varphi \varphi}$ & $12.46 \pm 0.22^{\#}$ \\
\hline MY41g + Indo & 250 & 5 & $2.63 \pm 0.11$ & $13.63 \pm 0.51^{* * * \# \# \# \varphi \varphi \varphi}$ & $4.75 \pm 0.28^{\# \# \# \varphi \varphi \varphi}$ & 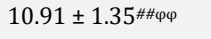 \\
\hline Suralfate + Indo & 50 & 5 & $2.38 \pm 0.19$ & 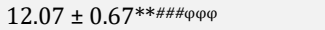 & $3.74 \pm 0.20^{\# \varphi}$ & 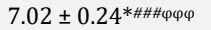 \\
\hline
\end{tabular}

Control 1 (4 day ulcerated rats); Control 2: (spontaneous healing in ulcerated rats without indomethacin) Control 3: (spontaneous healing in ulcerated rats given indomethacin). $N=$ number of rats; the values in the table represent averages $\pm E S M ;{ }^{*} p<0.05 ;{ }^{* *} p<0.01$; and ${ }^{* * *} p<0.001$ : Statistically significant compared to Control $1 ; \# p<0.05 ; \# \# p<0.01$ and

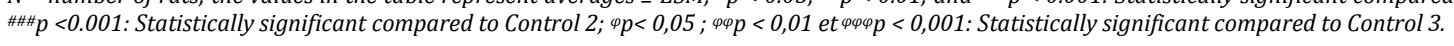

The ulcer indices decreased from 24.50 and $37.50 \mathrm{~mm}^{2}$ in spontaneous healing in ulcerated rats without and with indomethacin respectively, to 7.75 and $6.00 \mathrm{~mm}^{2}$ in rats treated with MY41g clay at 125 and $250 \mathrm{mg} / \mathrm{kg}$, respectively $(\mathrm{p}<0.001)$, with healing rates of 89.23 and $91.66 \%$ respectively (Table 4). Administration of MY41g clay at 125 and 250 $\mathrm{mg} / \mathrm{kg}$ caused in a significant decrease $(\mathrm{p}<0.001)$ in gastric acidity compared to the cross-sectional and longitudinal + indometacin controls. This gastric acidity value decreased from 73.75 in the cross-sectional control to 36.25 and 21.25 in rats treated with MY41g clay at 125 and 250 $\mathrm{mg} / \mathrm{kg}$, respectively. The significance of gastric acidity observed previously was same when animals were treated with sucralfate (Table 5).

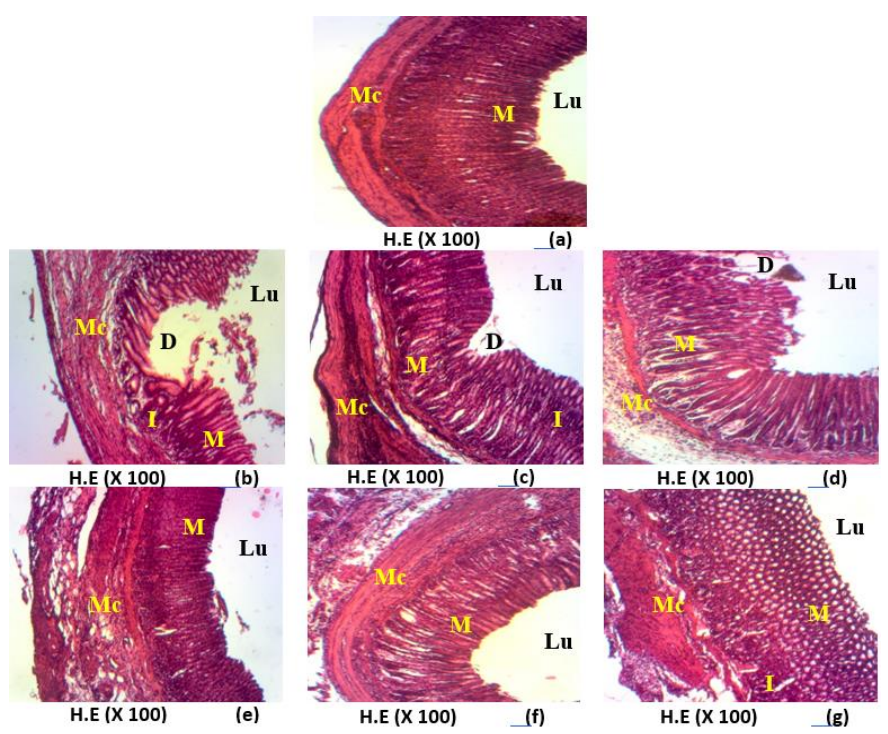

Fig. 4 Histological presentation of "unhealed gastric ulcers". 4(a): normal rat (shows normal muscle and mucosa); 4(b) : control 1 (shows destruction of the mucosa with lymphocyte infiltration); 4 (c and d) : controls 1 and 2 (show signs of self-healing, with an onset of mucosal regeneration but with more extensive mucosal destruction in the control 2); 4(e and f) : 125 and $250 \mathrm{mg} / \mathrm{kg}$ of MY41g clay; (progressive restoration of the mucosa with reduction of the inflammatory zone); (g) : $50 \mathrm{mg} / \mathrm{kg}$ sucralfate (shows normalization of the mucosa); D: destruction; E: edema; H.E: HematoxylinEosin; I: Leukocyte infiltration; Lu: Gastric lumen; M: mucosa; Mc: Muscle layer https://doi.org/10.30799/jpmr.048.20050103
Histological sections of "unhealed gastric ulcers" are shown in Fig. 4. The histological sections of the stomachs of rats in the normal and control 1 (4-day ulcerated rats) groups (Figs. 4(a) and (b)) are the same as those described above.

In control 2 (spontaneous healing in ulcerated rats without indomethacin), the section (Figs. 4(c) and (d)) shows signs of self-healing, and the onset of mucosal regeneration can be seen. MY41g clay (125 $\mathrm{mg} / \mathrm{kg}$ and $250 \mathrm{mg} / \mathrm{kg}$ ) resulted in progressive muscular restoration with decreased inflammatory zone compared to controls 1,2 and 3 (spontaneous healing in ulcerated rats with indomethacin) (Figs. 4(e) and (f), respectively). Histological section of rats treated with sucralfate shows normalization of the mucosa, but inflammation can still be perceived by the presence of lymphocyte infiltration zone (Fig. 4(g)).

The effects of MY41g clay on some parameters of oxidative status are shown in Table 6 . Indomethacin ip injection (1 mg/kg) for 14 days induced in rats an increase in malondialdehyde (MDA; $13.20 \mathrm{pmol} / \mathrm{mg}$ protein) and a reduction in superoxide dismutase (SOD; $1.06 \mathrm{U} / \mathrm{mg}$ protein) activity, reduced glutathione (GSH; $2.14 \mathrm{mmol} / \mathrm{g}$ protein) and catalase (CAT; 8.25 $\mu \mathrm{mol} \mathrm{H}_{2} \mathrm{O}_{2} / \mathrm{min} / \mathrm{mg}$ protein) compared to control 3 (spontaneous healing in ulcerated rats with indomethacin) (MDA: $14.11 \mathrm{pmol} / \mathrm{mg}$ protein; SOD: $1.90 \mathrm{U} / \mathrm{mg}$ protein; GSH: $2.20 \mathrm{mmol} / \mathrm{g}$ protein and CAT: $6.44 \mu \mathrm{mol}$ $\mathrm{H}_{2} \mathrm{O}_{2} / \mathrm{min} / \mathrm{mg}$ protein). Administration of MY41g clay concomitantly with indomethacin for 14 days resulted in a significant decrease in MDA levels $(12.46(\mathrm{p}<0.05)$ and $10.91(\mathrm{p}<0.01)$, respectively) in rats at 125 and 250 $\mathrm{mg} / \mathrm{kg}$; and a significant increase $(\mathrm{p}<0.001)$ in GSH levels of 5.23 and 4.75 $\mathrm{mmol} / \mathrm{g}$ protein, respectively, and Catalase $(\mathrm{p}<0.001)$ of 12.89 and 13.63 compared to controls. These same parameters (MDA, GSH, Catalase and SOD) varied in a similar way in animals treated with sucralfate.

The in vitro anti-acid capacity of MY41g clay demonstrated by Banenzoue et al. [7], sparked the interest that led us to conduct an in vivo study of the curative and antacid effects of the clay solution on chronic and "unhealed" gastric ulcers. In replacement of the $\mathrm{CaCO}_{3}$ used in the in vitro study, we used a local material (limestone) to improve the buffering capacity of the MY41g clay. In this study, the healing potential of clay was evaluated firstly on acetic acid induced chronic gastric ulcers which bear a high resemblance to human ulcers. In addition, the second experiment simulated the real case of elderly patients who, while treating chronic gastric ulcers, are often required to take NSAIDs for the relief of pain associated with other age-related conditions like arthritis and rheumatism. NSAIDs have been shown to retard the healing of gastric ulcers, leading to the so called "unhealed" or "hard healing" ulcers. 
The injection of acetic acid into the stomach walls of rats produces ulcers that are pathologically and therapeutically similar to human gastric ulcers [24]. The development of these ulcers is due to the action of acetic acid, which corrodes the layers of the gastric wall and causes the acidity of the gastric juice to increase by gastric obstruction $[25,26]$. These ulcers are also induced by the stress caused by the laparotomy performed during ulcer induction. Through physiological and psychological factors (decreased gastric blood flow, increased gastric motility, vagal hyperactivity), stress promotes a significant accumulation of acid and pepsin in the stomach lumen. The result is tissue necrosis that causes the release of arachidonic acid metabolites; this attracts leukocytes (neutrophils and macrophages), resulting in the transformation of superficial lesions into deeper wounds; and inactivation of growth factors important to maintaining mucosal integrity and repair [27]. The high ulcer index $\left(72.00 \mathrm{~mm}^{2}\right)$ observed in ulcerated rats 4 days after ulcer induction is the result of this pathophysiological mechanism of acetic acid ulcer induction.

In the chronic ulcer induction model with acetic acid, ulcerated rats given distilled water only during the treatment period showed $71.18 \%$ self-healing. This healing is justified by the fact that tissue necrosis causes the release of arachidonic acid metabolites, which attracts leukocytes (neutrophils and macrophages). These leukocytes phagocyte necrotic tissue and release pro-inflammatory cytokines and growth factors that activate fibroblasts, endothelial cells and epithelial cells. This activation is at the origin of the formation of the granulation tissue (tissue replacing the damaged area) [28]. However, human patients with chronic gastric ulcers, can't rely on self-healing and treatment should therefore be combined with the elimination of etiological factors.

The MY41g clay solution, administered once a day for 10 days per os, significantly accelerated the spontaneous healing of chronic gastric ulcers. This acceleration of spontaneous healing was accompanied by a significant increase $(\mathrm{p}<0.001)$ in mucus production. The importance of reinforcement of the gastric epithelium by increasing mucus production is well known [29]. Indeed, mucus is a glycoprotein that intervenes in the protective barrier of the gastric mucosa, forming an insoluble gel that adheres to the surface of the mucosa and prevents its destruction by aggressive substances [30]. The works of Tan et al., Amang et al. and Kuissu et al. [31-33] have shown that during the healing process, the aqueous extracts of Ocimum suave, Eremomastax. speciosa and Enantia chlorantha, respectively, increase mucus secretion, thus offering protection of the ulcer crater against gastric acid secretion and consequently hastening the healing process. In addition, Leonard et al. [34] showed that aluminium silicates inhibit the corrosive action of pepsin by increasing the thickness of the gastric mucus. MY41g clay could act in a similar way to accelerate the spontaneous healing process. Inflammation in general is characterized by intense neutrophil infiltration associated with vascular dilation. This is followed by the proliferation stage, which manifests itself in angiogenesis, collagen deposition, wound contraction and epithelialization. The subsequent remodeling phase consists of the formation of new collagen and an increase in the cohesive strength of the newly formed tissues $[35,36]$. Healing is a normal physiological process that takes place through a series of coordinated cellular events that culminates in the restoration of the anatomical and functional integrity of tissues [37]. Ulcer healing is a complex process that depends on the regeneration of the structure of the glandular mucosa and the migration of epithelial cells to the ulcer crater in order to cover it [38]. In this study, clay caused a decrease in the ulcerated area, with repair of the glandular epithelium. It is therefore obvious that MY41g clay promotes the healing of chronic ulcers by acting on one or more cellular and molecular processes involved in the healing process. The anti-inflammatory, healing and covering properties of MY41g clay are likely due to the mineralogical presence of kaolinite, whose content of copper (105 ppm) will stimulate immunity and have an anti-inflammatory effect [39]. Mahraoui et al. [40] showed that aluminium silicates can prevent cell disjunction induced by inflammatory cytokines. In fact, studies have shown that $\mathrm{SiO}_{2}(47.74 \%)$ and $\mathrm{Al}_{2} \mathrm{O}_{3}(35.54 \%)$ represent $80 \%$ of the minerals contained in the MY41g sample [41]. Thus, the mineralogical composition of MY41g clay could be exploited as an ideal healing and protective dressing for the mucous membranes of the digestive tract $[42,43]$.

It is known that; non-steroidal anti-inflammatory drugs have a negative impact on ulcer healing [38]. This fact has been verified by Wang et al., Amagase et al. and Amang et al. [16, 44, 45], who concluded that repeated administration of indomethacin for 14 days significantly delays spontaneous healing of gastric ulcers induced with acetic acid, creating "unhealed gastric ulcers". This was confirmed in the present study when the degree of self-healing reduced from $65.97 \%$ in ulcerated rats given no NSAID to $47.91 \%$ in ulcerated rats given indomethacin. Indeed, the pathogenesis of gastric lesions associated with the administration of indomethacin is related to the non-selective and irreversible inhibition of cyclooxygenases 1 and 2 [46]. This inhibition prevents the synthesis of prostaglandins (prostacyclin $\left(\mathrm{PGI}_{2}\right)$ and prostaglandin $\mathrm{E} 2\left(\mathrm{PGE}_{2}\right)$ ) which have protective functions in the gastric mucosa. Indeed, $\mathrm{PGI}_{2}$ via these IP receptors is responsible for relaxation of vascular smooth muscles of the gastric microcirculation, by increasing the production of intracellular cAMP. Prostaglandins $\left(\mathrm{PGE}_{2}\right)$ and prostacyclins $\left(\mathrm{PGI}_{2}\right)$ are two powerful vasodilators that, once bound to the $\mathrm{EP}_{3}$ and IP receptors, control almost all aspects of defense and healing (the secretion of bicarbonates in mucosal cells; it decreases gastric acid secretion directly on parietal cells by decreasing intracellular cAMP concentration and indirectly by inhibiting histamine release from the gastric mucosa) [47]. In addition, $\mathrm{PGE}_{2}$ has been shown to be a potent inhibitor of the release of tumor necrosis factor (mast cells and macrophages), platelet activation factor (mast cells), interleukin-1 (macrophages), leukotrienes (neutrophils) and interleukin-8 (neutrophils) which are all pro-inflammatory mediators [48]. In addition, indomethacin will cause the expression of intercellular adhesion molecules (ICAM-1) responsible for neutrophil adhesion [48] They pile up in the microcirculation, causing a local decrease in the blood flow of the mucous membrane which increases vascular tone, exacerbates tissue ischemia, stimulate the production of reactive oxygen species and thus lead to a severe degree of necrosis, particularly in the presence of a low luminal $\mathrm{pH}$ [45]. Indomethacin also reduces the activity of antioxidant enzymes such as superoxide dismutase (SOD), catalase (CAT), and reduced glutathione (GSH) [49]. The role of these enzymes in the body's defense against oxidative stress is well known [50]. All these phenomena would explain the significant damage observed in the ulcerated animals concomitantly treated with indomethacin.

Macromorphologically, clay treatment at doses of 125 and $250 \mathrm{mg} / \mathrm{kg}$ resulted in a significant reduction in the ulcer index. The antiinflammatory properties of this clay could be an effective weapon in the fight against indomethacin in its mobilizing effects of the above-mentioned pro-inflammatory factors and would therefore explain the decrease in the percentage ulcerated surface. In addition, it was observed that kaolinites, smectites and attapulgite clays stimulate coagulation factors in vitro [51]. Tarnawski et al. showed that re-epithelialization of the ulcerated mucosa is an essential process for the healing of gastrointestinal ulcers, and without the continuous restoration of an epithelial barrier, the mucosa would be vulnerable to infections and lesions of mechanical or chemical origin [52]. Significant promotion of mucus production allows clays to solve the major problem of indomethacin which is to inhibit prostaglandin production and would thus contribute to accelerate the spontaneous healing of these ulcers. Gwozdinski et al. showed that the binding of clay crystals to mucus improves its rheological characteristics (viscosity, hydrophobia, polymerization of glycoproteins and adhesion to the wall of the digestive tract) and reduces its degradation [53]. Mucus has a dual protective role: physical protection by acting as a lubricant for the mucosa while preventing direct contact between acidic gastric juice and the epithelium of the gastric mucosa [54], thus promoting the proper progress of the healing process; chemical protection against the proteolytic and acidic properties of gastric juice by sequestering bicarbonate, creating a $\mathrm{pH}$ gradient between gastric juice and epithelium of the gastric mucosa [55 54]. In addition, kaolinites have been shown to prevent and treat digestive diseases by healing the stomach and intestinal mucosa, by improving mucus production, stopping micro-bleeds, and cauterizing digestive ulcers [55].

The effects of MY41g clay, and sucralfate were better visualized on the histological sections of the stomach tissue of each group. Ulcerated rats given indomethacin showed more destruction of the mucosa than the simple longitudinal control. This would be due to the action of indomethacin, which would slow down the process of spontaneous healing. Severe fibrosis, persistent neutrophil infiltration, interference with the action of growth factors, slowing of angiogenesis at the base of the ulcer and maturation of granulation tissue are processes involved in the induction mechanism of "unhealed gastric ulcers" [16]. Although the ulcerated rats given indomethacin showed destruction of the mucosa, it also showed with the ulcerated rats without indomethacin a restoration of the musculature compared to 14 days ulcerated rats, which would be the result of the self-healing process, put in place by the body. MY41g clay as well as sucralfate caused a progressive and improved reconstitution of the mucosa and muscular system. The effectiveness of MY41g clay could be due to the fact that, in addition to being an anti-inflammatory, it strengthens the mucobicarbonate barrier. The secretion of gastric acid in the stomach also plays an important role in delaying the healing of gastric ulcers. This acid interferes with gastric mucosal restoration processes, resulting in the conversion of superficial lesions into deeper mucosal lesions, and the inactivation of growth factors important for maintaining mucosal integrity and repairing gastric lesions. In both ulcer models studied, there was a significant decrease in gastric acidity in the MY41g clay-treated groups compared to 14 days ulcerated rats and in ulcerated 
rats without and with indomethacin groups. The clay solution could therefore neutralize the $\mathrm{H}^{+}$ions in the stomach lumen, and therefore increase the $\mathrm{pH}$ of the solution. Leonard et al. showed that clay captures pepsin and can therefore totally inhibit mucosal damage, hemorrhagic lesions and ulcerations usually created by excessive pathological secretion of pepsin [34]. Thus, this capacity of the clay solution to buffer the acidity could allow it to accelerate the spontaneous healing process.

Reactive oxygen species (ROS) are known to be involved in the genesis of gastric lesions [52]. Lipid peroxidation resulting from oxidative stress is a mechanism by which oxygenated free radicals cause tissue damage [56]. Oxidative stress thus causes cytotoxicity and inhibition of wound healing [57], while antioxidants help cells to protect them from damage due to oxidative stress [50].

In this study, concomitant administration of MY41g clay with indomethacin significantly prevented the increase in MDA levels, reverting them back to above the normal values. The significant reduction in MDA levels accompanied by a significant increase in GSH levels and catalase activity suggests a reduction in oxidative stress characterized by a decrease in lipid peroxidation and an increase in antioxidant capacities. The ability of MY41g clay to prevent the delayed healing of "unhealed gastric ulcers" may also be related to its antioxidant activity. Similar results have been observed by Amang et al. and Kuissu et al. $[45,33]$ with the aqueous extracts of Eremomastax. speciosa and Enantia chlorantha respectively, on "unhealed gastric ulcers".

\section{Conclusion}

Administration of MY41g clay accelerated the spontaneous healing of chronic acetic acid-induced gastric ulcers and prevented the delay in the healing of chronic gastric ulcers caused by indomethacin. The mode of action of the MY41g clay cool include: increased gastric mucus thickness, increased gastric reepithelialization, improved antioxidant status and effective antacid activity. The rich mineralogical composition of MY41g clay can be exploited for the development of an affordable replacement for other antacids in the triple therapy regimen for ulcer treatment.

\section{Acknowledgments}

The authors would like to thank the University of Yaoundé I and the institute of medical research and study of medicinal plants (IMPM) of Cameroon for the setting up of the technical platform during the realization of this work.

\section{References}

[1] W.H.O, General guidelines for methodologies on research and evaluation of traditional medicine, World Health Organization, Geneva, 2000.

[2] A. Lelief-Delcourt, Clay is smart, Ed Leducs., dépôt légal, Paris France, 2011.

[3] D.M. Moore, R.C. Reynolds, X-ray diffraction and the identification and analysis of clay minerals, 2nd Ed., Oxford University Press, England, 1997.

[4] R. Prost, Study of hydration of clays: water-mineral interactions and mechanism of water retention, II, Study of a smectite (hectorite), Int. Inf. Syst. Agric. Sci. Technol. 26 (2013) 263-535.

[5] W.H.O, WHO Strategy for traditional medicine, World Health Organization, Geneva, 2002.

[6] J. Allègre, Alumina silicates (clays) in therapeutics: an ancient customary practice relayed in modern medicine, Doctoral thesis in medicine, University of Paris XIII, France, 2012.

[7] G.I. Ekosse, Kaolin deposits and occurrences in Africa: Geology, mineralogy and utilization, Appl. Clay. Sci. 50 (2010) 212-236.

[8] C. Banenzoue, P. Signing, J.A. Mbey, D. Njopwouo, Antacid power and their enhancements in some edible clays consumed by geophagia in Cameroon, J. Chem. Pharm. Res. 6(10) (2014) 668-676.

[9] M.I. Grossman, Peptic ulcer: A guide for the practicing physician, Year Book Medical Publishers, United States, 1981

[10] M. Fornai, L. Antonioli, R. Colucci, M. Tuccori, C. Blandizzi, Pathophysiology of gastric ulcer development and healing: molecular mechanisms and novel therapeutic options, in: C. Jianyuan (Eds), peptic ulcer disease, InTech., China, 2011, pp.114-133.

[11] A. Njoya, C. Nkoumbou, C. Grosbois, D. Njopwouo, D. Njoya, et al., Genesis of Mayouom kaolin deposit (Western Cameroon), Appl. Clay. Sci. 32 (2006) 125140.

[12] Council of Europe, European convention for the protection of vertebrate animals used for experimental and other scientific purposes, European Treaty Series no. 123, Strasbourg 18 III, 1986.

[13] N.R. Pillai, G. Santhakumari, Effects of nimbidin on acute and chronic gastroduodenal ulcer models in experimental animals, Planta. Med. 50(2) (1984) 143-146.

[14] N. Hara, S. Okabe, Effect of gefarnate on acute lesions in rats, Fol. Pharmacol. Jpn. 85 (1985) 443-448.

https://doi.org/10.30799/jpmr.048.20050103
[15] P.V. Tan, B. Nyasse, G.E. Enow-Orock, P. Wafo, E.A. Forcha, Prophylactic and healing properties of a new anti-ulcer compound from Enantia chlorantha in rats, Phytomed. 7(4) (2000) 291-296.

[16] J.Y. Wang, S. Yamasaki, K. Takeuchi, S. Okabe, Delayed healing of acetic-induced gastric ulcers in rats by indomethacin, Gastroenterol. 96 (1989) 393-402.

[17] P.V. Tan, N.G. Nditafon, M.P. Yewah, T. Dimo, F.J. Ayafor, Eremomastax speciosa Effects of leaf aqueous extract on ulcer formation and gastric secretion in rats, J. Ethnopharmacol. 54(2-3) (1996) 139-142.

[18] F. Bayelet-Vincent, Cytology and pathology records technical, Bayer., Diagnostics, London, 2002.

[19] R.J. Henry, D.C. Canon, J.W. Winkelman, Clinical chemistry, principles and Techniques, 2nd Ed., Harper and Row, Hagerstown, Maryland, 1974.

[20] G.L. Ellman, Tissue sulfhydryl groups, Arch. Biochem. Biophys. 82(1) (1959) 70-77.

[21] E. Marhuenda, M.J. Martin, C.A. de La Lastra, Antiulcerogenic activity of aescine in different experimental models, Phytother. Res. 7(1) (1993) 13-16.

[22] A.K. Sinha, Colorimetric assay of catalase, Anal. Biochem. 47 (1972) 389-394.

[23] K.M. Wilbur, F. Bernheim, O.W. Shapiro, Determination of lipid peroxidation, Arch. Biochem. Biophys. 24 (1949) 305-310.

[24] S. Okabe, K. Amagase, An overview of acetic acid ulcer models--the history and state of the art of peptic ulcer research, Biol. Pharmacol. Bull. 28(8) (2005) 1321-1341.

[25] A. Tarnawski, Cellular and molecular mechanisms of gastrointestinal ulcer healing, Dig. Dis. Sci. 50 (2005) 24-33.

[26] V. Molina, D. Carbajal, M. Noa, Y. Ravelo, R. Mas, M. Valle, Comparative effects of D -002, Ranitidine and Omeprazole on acetic acid-induced ulcers, Int. J. Pharm. Sci. 5(4) (2013) 91-95.

[27] M. Jainu, S.S.D. Chennan, Antiulcerogenic and ulcer healing effects of Solanum nigrum (L.) on experimental ulcer models: possible mechanism for the inhibition of acid formation, J. Ethnopharmacol. 104 (2005) 156-168.

[28] L. Laine, K. Takeuchi, A. Tarnawski, Gastric mucosal defense and cytoprotection: bench to bedside, Gastroenterol. 135(1) (2008) 41-60.

[29] C.M. Polo, T.M. Moraes, C.H. Pellizzon, M.O. Marques, L.R.M. Rocha, Hiruma C.A Lima, Gastric ulcers in middle-aged rats: The healing effect of essential oil from Citrusaurantium L. (Rutaceae), Evid-based. Complement. Altern. Med. 10 (2012) 1-8.

[30] E. Gaudier, C. Hoebler, Physiological role of mucins in the colonic barrier integrity, Gastroenterol. Clin. Biol. 30 (2006) 965-974.

[31] P.V. Tan, C. Mezui, G.E. Enow-Orock, G. Agbor, Antioxidant capacity, Cytoprotection, and healing actions of the leaf aqueous extract of Ocimum suave in rats subjected to chronic and cold-restraint stress ulcers, BioMed. Res. Int $10(2013) 1-9$.

[32] A.P. Amang, P.V. Tan, A.S. Patamaken, N.M. Mefe, Cytoprotective and antioxidant effects of the methanol extract of Eremomastax speciosa in rats complement alternative medicine, Afr. J. Tradit. Med. 11(1) (2014) 165-171.

[33] T.M.M. Kuissu, P.V. Tan, E.G. Enow-Orock, C. Mezui, Z.E. Nkwengoua, B. Nyasse Aqueous extract of Enantia chlorantha (Annonaceae) prevents the delay in chronic gastric ulcer healing caused by indomethacin in rats, Br. J. Pharmacol. Res. 8(1) (2015) 1-13.

[34] A. Leonard, M.T. Droy-Lefaix, A. Allen, Pepsin hydrolysis of the adherent mucus barrier and subsequent gastric mucosal damage in the rat : effect of diosmectite and 16 dimethyl prostaglandin E2, Gastroenterol. Clin. Biol. 18 (1994) 609-616.

[35] S. Shetty, S. Udupa, L. Udupa, Evaluation of antioxidant and wound healing effects of alcoholic and aqueous extracts of Ocimum sanctum in rats, Evid-Based Complement. Altern. Med. 5 (2008) 95-101.

[36] P.N. Badri, S. Renu, Role of medicinal plants in wound healing, Res. J. Med. Pl. 5 (2011) 392-405

[37] M.R. Anamika, S. Arti, Antioxidant status in delayed healing type of wounds, Int. J. Exp. Pathol. 8(4) (2000) 257-263.

[38] L. Shawon, P. Gautam, An overview of the current methodologies used for evaluation of gastric and duodenal anti-ulcer agents, Pharmacol. 3(8) (2012) 249-257.

[39] T.C. Pereira, M.M. Campos, M.R. Bogo, Copper toxicology, Oxidative stress and inflammation using zebrafish as experimental model, J. Appl. Toxicol, 36(7) (2016) 876-85.

[40] L. Mahraoui, M. Heyman, O. Plique, M.T. Droy-Lefaix, J.F. Desjeux, Apical effect of diosmectite on damage to the intestinal barrier induced by basal tumour necrosis factor alpha, Gastroenterol. 40(3) (1997) 339-343.

[41] T.P. Boulleys, Contribution to the physico-chemical characterization of clays from Mayouom: Case of wells 3 and 4. Post Graduate Diploma Dissertation, University of Yaoundé I, Yaoundé, Cameroon, 2005.

[42] P. Chavanne, 200 clay remedies, 1 st Ed., Legal deposit, France, 2011.

[43] N. Cousin, Clay, Ed Eyrolles, Legal deposit, France, 2013.

[44] K. Amagase, M. Yokota, Y. Tsukimi, S. Okabe, Characterization of 'unhealed gastric ulcers' produced with chronic exposure of acetic acid ulcers to indomethacin in rats, J. Physiol. Pharmacol. 54(3) (2003) 349-360.

[45] A.P. Amang C. Mezui, G.T. Siwe, J. Emakoua, G. Mbah, et al, Healing and antisecretory effects of aqueous extract of Eremomastax speciosa (Acanthaceae) on unhealed gastric ulcers, BioMed. Res Int. 10 (2017) 1-11.

[46] C. Musumba, D.M. Pritchard, M. Pirmohamed, Review article: cellular and molecular mechanisms of NSAID-induced peptic ulcers, Aliment. Pharmacol. Ther. 30 (2009) 517-531.

[47] J. L. Wallace, Prostaglandines, NSAIs and gastric mucosal protection: why doesn"t the stomach digestive itself, Physiol. Rev. 88 (2008) 1547-1565.

[48] A.Z.A. Inas, K.A.H. Hala, H.H. Gehan, Gastroprotective effect of Cordia myxa L. fruit extract against indomethacin-induced gastric ulceration in rats, Life. Sci. J. 8(3) (2011) 433-445.

[49] V. Mollace, C. Muscoli, E. Masini, S. Cuzzocrea, D. Salvemini, Modulation of prostaglandin biosynthesis by nitric oxide and nitric oxide donors, Pharmacol. Rev. 57 (2005) 212-252. 
[50] M.A. Abdulla, F.H. AL-Bayaty, L.T. Younis, H.M.I. Abu, Antiulcer activity of Centella asiatica leaf extract against ethanol-induced gastric mucosal injury in rat, J. Med. Pl. Res. 4(13) (2010) 1253-1259.

[51] D. Long, Clays in veterinary therapeutics, Veterinary Medicine Thesis, Lyon, France, 2002

[52] A. Tarnawski, I.L. Szabo, S.S. Husain, B. Soreghan, Regeneration of gastric mucosa during ulcer healing is triggered by growth factors and signal transduction pathways, J. Physiol. 95(1-6) (2001) 337-344.

[53] K. Gwozdinski, A. Jedrzejewska, M. Janocka, M.T. Droy-Lefaix, Effect of diosmectite on the physico-chemical properties of gastric mucus in vivo and in vitro, Gastroenterol. 12 (1997) 136:1-4.
[54] W. Lippincott, Wilkins, High-yield Physiology, $1^{\text {st }}$ Ed., Philadelphie, 2007.

[55] S.L. Simon, Soil ingestion by humans: a review of history, data and etiology with application to risk assessment of radioactively contaminates soil, Health. Physiol. 74 (1998) 647-672.

[56] S. Sen, R. Chakraborty, B. Debnath, J. Mazumder, Plants and phytochemicals for peptic ulcer: An overview, Pharmacol. Rev. 3(6) (2009) 270-279.

[57] J. Dissemond, M. Goos, S.N. Wagner, The role of oxidative stress in pathogenesis and therapy of chronic wound, Hautarzt. 3(11) (2002) 718-723. 